\title{
A neutral-niche theory of nestedness in mutualistic networks
}

\author{
Abhay Krishna, Paulo R Guimarães Jr, Pedro Jordano and Jordi Bascompte \\ A. Krishna (abhay@ebd.csic.es), P. R. Guimarāes Jr, P. Jordano and J. Bascompte, Integrative Ecology Group Estación Biológica de Doňana, \\ CSIC Apdo. 1056, ES-41080 Sevilla, Spain. Present address for PRG: Instituto de Fisica, Gleb Wataghin UNICAMP, 6109, BR-13083-970 \\ Campinas, SP, Brazil.
}

\begin{abstract}
Recently, there has been a vigorous interest in community ecology about the structure of mutualistic networks and its importance for species persistence and coevolution. However, the mechanisms shaping mutualistic networks have been rarely explored. Here we extend for the first time the neutral theory of biodiversity to a multi trophic system. We focus on nestedness, a distinctive pattern of mutualistic community assembly showing two characteristics, namely, asymmetrical specialization (specialists interacting with generalists) and a generalist core (generalists interacting with generalists). We investigate the importance of relative species abundance (RSA) for the nested assembly of plant-animal mutualistic networks. Our results show that neutral mutualistic communities give rise to networks considerably more nested than real communities. RSA explains $60-70 \%$ of nested patterns in two real communities studied here, while $30-40 \%$ of nestedness is still unexplained. The nested pattern in real communities is better explained when we introduce interactionspecific species traits such as forbidden links and intensity of dependence (relative importance of fruits for the diet of a frugivore) in our analysis. The fact that neutral mutualistic communities exhibit a perfectly nested structure and do not show a random or compartmentalized structure, underlines the importance of RSA in the assembly of mutualistic networks.
\end{abstract}

Recent studies demonstrate that mutualisms among free living species often form nested networks, i.e. those species with fewer interactions are preferentially associated with a subset of species that interact with the most connected ones (Bascompte et al. 2003, Dupont et al. 2003, Ollerton et al. 2003, 2007, Guimarães et al. 2006, 2007). Nestedness is characterized by asymmetrical interactions (specialists interacting with generalists) and a generalist core (generalists interacting with generalists) (Bascompte et al. 2003).

In the quest of an explanation for nested structure, it has been suggested that nestedness may emerge as a consequence of the combined effects of species abundance (Jordano 1987, Dupont et al. 2003, Vázquez et al. 2005, Jordano et al. 2006, Lewinsohn et al. 2006), coevolutionary geographic mosaics of interaction (Thompson 2005, Guimarães et al. 2006), alignment of phenotypic traits (Stang et al. 2006, Rezende et al. 2007a, Santamaria and Rodriguez-Girones 2007), or past evolutionary history (Rezende et al. 2007b). However no previous study develops a theoretical framework to quantify the importance of relative species abundance (RSA) to nestedness. Here we investigate the effects of RSA on nestedness in mutualistic assemblages by combining theory and empirical data (independent observations of local abundance and interaction frequency; relative importance of fruits in diet of frugivores). We attempt for the first time to connect the nested assemblage of mutualistic networks to individual based processes by taking RSA into account using the neutral theory of biodiversity (Bell 2001, Hubbell 2001).

The neutral theory of biodiversity aims to explain the diversity and relative abundance of species in a single trophic community. The fundamental assumption of neutral theory is that all species in a single trophic level are identical in their fitness and in their effects on other species sharing the same trophic level. The only driver of population dynamics in such unitrophic neutral communities is random variation in birth, death and dispersal. Neutral theory predicts a dispersal limited multinomial (DLM) distribution of species abundance (Hubbell 2001, Etienne et al. 2007) with a long tail of rare species (singletons).

We extend the neutral theory of biodiversity for a single trophic community to generate neutral mutualistic networks, in which interactions are determined by DLM species abundance distributions as predicted by the neutral theory (Hubbell 2001) for each trophic level. We compared the results for these neutral mutualistic networks with the analyses of two real plant-frugivore interaction networks for which an independent estimate of RSA is available. Finally, we show through numerical simulations and real field data analysis, that the effects of abundance (neutral processes) are modulated by interaction-specific species traits such as forbidden links (Jordano et al. 2003) and the intensity of frugivory (Jordano and Schupp 2000). We have used the neutral model to generate SAD of mutualistic partners and 
we use two null models as described below, to compare the networks (real and neutral) of varying sizes and connectivity, using the metrics of relative nestedness (details about the differences and similarities between neutral and null models are described in Gotelli and McGill 2006).

We show here that nested patterns in real mutualistic networks appear to be better explained by a process that combines neutral and interaction-specific, species traits. As shown below, we also compare the relative nestedness of a large data set of mutualistic webs with neutral mutualistic networks having different frequencies of forbidden links, in an effort to generalize the effect of modulating factors on abundance-generated nestednes. The fact that neutral mutualistic communities exhibit perfectly nested structure and do not show a random or compartmentalized structure, underlines the importance of RSA in the assembly of mutualistic networks.

\section{Material and methods}

\section{Neutral mutualistic networks}

We generated RSA distributions for plant and animal mutualists separately using the associated sequential urn scheme of Etienne's new sampling formula of neutral biodiversity (Etienne and Olff 2004, Etienne 2005):

$$
\begin{aligned}
\operatorname{Pr} & \left(\mathrm{n}_{1}, \mathrm{n}_{2}, \ldots, \mathrm{n}_{\mathrm{s}} \mid \theta, \mathrm{m}, \mathrm{J}\right) \\
= & \frac{\mathrm{J} !}{\Pi_{\mathrm{i}=1}^{\mathrm{s}}\left(\mathrm{n}_{\mathrm{i}}\right) \Pi_{\mathrm{j}=1}^{\mathrm{J}}\left(\Phi_{\mathrm{j}} !\right)} \frac{\theta^{\mathrm{s}}}{(\mathrm{I})_{\mathrm{J}}} \sum_{\mathrm{A}=\mathrm{s}}^{\mathrm{J}} \mathrm{K}(\overrightarrow{\mathrm{D}}, \mathrm{A}) \frac{\mathrm{I}^{\mathrm{A}}}{(\theta)_{\mathrm{A}}}
\end{aligned}
$$

This formula is used to evaluate the likelihood of neutral theory parameters given a species abundance distribution (SAD). The associated sequential urn scheme (Etienne and Olff 2004, Etienne 2005) generates probability distribution samples of a community of $\mathrm{J}$ individuals composed of $s$ distinct species with abundance $\mathrm{n}_{1}$ for the species $1, \mathrm{n}_{2}$ for the species 2 and so on up to $n_{s}$ for the species $s$ with an assumption that the total number of individuals in the community always remains the same (zero sum assumption), but see Etienne et al. (2007). $\mathrm{m}$ is dispersal probability, $\theta$ is the fundamental biodiversity number and depends on the number of individuals in the community $(\mathrm{J})$ and speciation rate $(v) ; \theta=2 \mathrm{Jv} ; \Phi_{\mathrm{j}}$ is the number of species that have abundance $j$ and $I$ is the number of immigrants that compete with the local individuals for vacant spots in a local community:

$\mathrm{I}=(\mathrm{J}-1) \frac{\mathrm{m}}{1-\mathrm{m}}$

$\mathrm{K}(\overrightarrow{\mathrm{D}}, \mathrm{A})$ represents the sum of all possible species-ancestryabundance datasets compatible with a species abundance distribution, and can be written as follows

$\mathrm{K}(\overrightarrow{\mathrm{D}}, \mathrm{A})=\sum_{\mathrm{a}_{1}, \ldots \mathrm{a}_{\mathrm{s}} \mid \sum_{\mathrm{i}=1}^{s} a_{\mathrm{i}}=\mathrm{A}} \Pi_{\mathrm{i}=1}^{S} \frac{\bar{s}\left(\mathrm{n}_{\mathrm{i}}, \mathrm{a}_{\mathrm{i}}\right) \bar{s}\left(\mathrm{a}_{\mathrm{i}}, 1\right)}{\bar{s}\left(\mathrm{n}_{\mathrm{i}}, 1\right)}$

For more details on $\mathrm{K}(\overrightarrow{\mathrm{D}}, \mathrm{A})$, see Etienne (2005) and Etienne and Olff (2004). After generating plant and animal RSA distributions separately, for $\mathrm{J}$ equal to 1000 , and different values of $\mathrm{m}(0.001,0.01,0.1,0.5,1)$ and $\theta(1,10$,
$25,50,100)$, we let these distributions interact. We created the interaction probability matrix (P) (Fig. 1) in which an interaction probability (between ith plant $\left(\mathrm{p}_{\mathrm{i}}\right)$ and $\mathrm{jth}$ animal $\left(a_{\mathfrak{j}}\right)$ is defined as:

$P\left(p_{i}, a_{j}\right)=\frac{J_{i}^{p}}{J^{P}} \frac{J_{j}^{a}}{J^{A}}$

where $\mathrm{J}_{1}^{\mathrm{P}}$ is the number of individuals of plant species $\mathrm{i}, \mathrm{J}_{j}^{\mathrm{a}}$ is the number of individuals of animal species $j, J^{P}$ is the number of individuals in the plant community and $\mathrm{J}^{\mathrm{A}}$ is the number of individuals in the animal community.

The final adjacency matrix which denotes a neutral mutualistic network was obtained by assuming that all plant and animal species have at least one interaction. The presence of an interaction $\left(a_{i j}=1\right.$, in the adjacency matrix $)$ between species $i$ and $j$ was determined by ranking the elements of the probability matrix $\mathrm{P}$ and setting an absence of interaction $\left(\mathrm{a}_{\mathrm{ij}}=0\right.$, in the adjacency matrix $)$ for those elements with $\mathrm{P}\left(\mathrm{p}_{\mathrm{i}}, \mathrm{a}_{\mathrm{j}}\right)$ below a certain cutoff value. The conservative estimate of cutoff value was based on the assumption that all plant and animal species have at least one interaction, i.e. the value of $\mathrm{P}\left(\mathrm{p}_{\mathrm{i}}, \mathrm{a}_{\mathrm{j}}\right)$, when the criterion of at least one interaction for all plants and animal species is met is considered as the cut-off value.

For example, if our community consist of four plants and four animals of varying abundances, we will have an interaction probability matrix $(4 \times 4)$ having probability of interaction based on RSAs. This probability matrix will give rise to the binary interaction matrix $(4 \times 4)$, in which all interaction probabilities high enough are one. Once, the species with lowest probabilities of interaction also have at least one interaction (say, species 4 of animals with least abundance is now interacting with species 1 of plants which is most abundant), we take a snapshot of interaction structure and stop converting probabilities to 1 . All lower probabilities are as good as no interaction (say, species 4 of animal will not interact with least abundant plant species 4).

Using the above method we generated 20000 neutral mutualistic networks (NMN); $800 \mathrm{NMN}$ for each pair of values of $m$ and $\theta$, with plant and animal communities having the same values of $m$ and $\theta$ (changing neutral theory parameters for plant and animal communities did not seem to change the results qualitatively).

In this model interaction structure is a secondary phenomenon of the coexistence of the two groups and the population dynamics is solely regulated by the competitive interactions among two groups. This implies that this model does not take into account the demographic consequences of the interaction structure, or interaction structure feeding back on abundance, that manifests itself at scales larger than the local community. There is a perfect symmetry at the individual level in each mutualistic event i.e., at the individual level each mutualistic individual has exactly the same effect on each other. Though here we concentrated on nestedness, yet it is worth to state that the connectance (ratio of number of realized interaction to the total possible interactions) in such neutral mutualistic webs show values as observed towards the high end of real mutuahstic webs $(0.12-0.4)$. The lower end of this connectance range $(0.12-0.25)$ is shown by neutral mutuahstic webs with a larger number of species (115-425) 

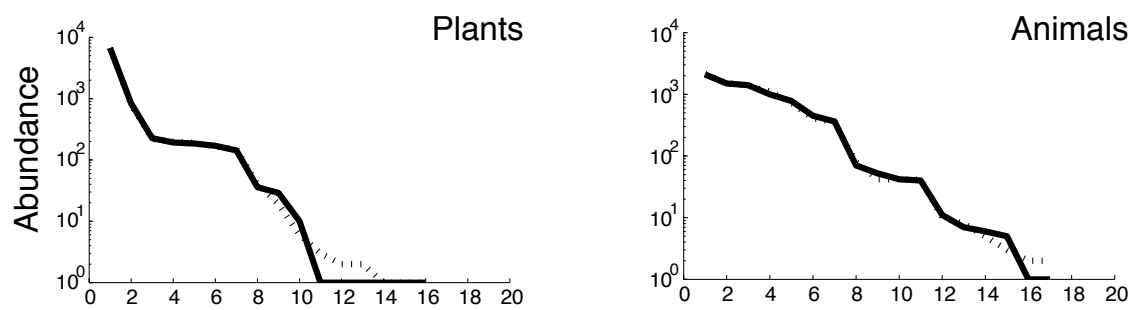

Rank in abundance

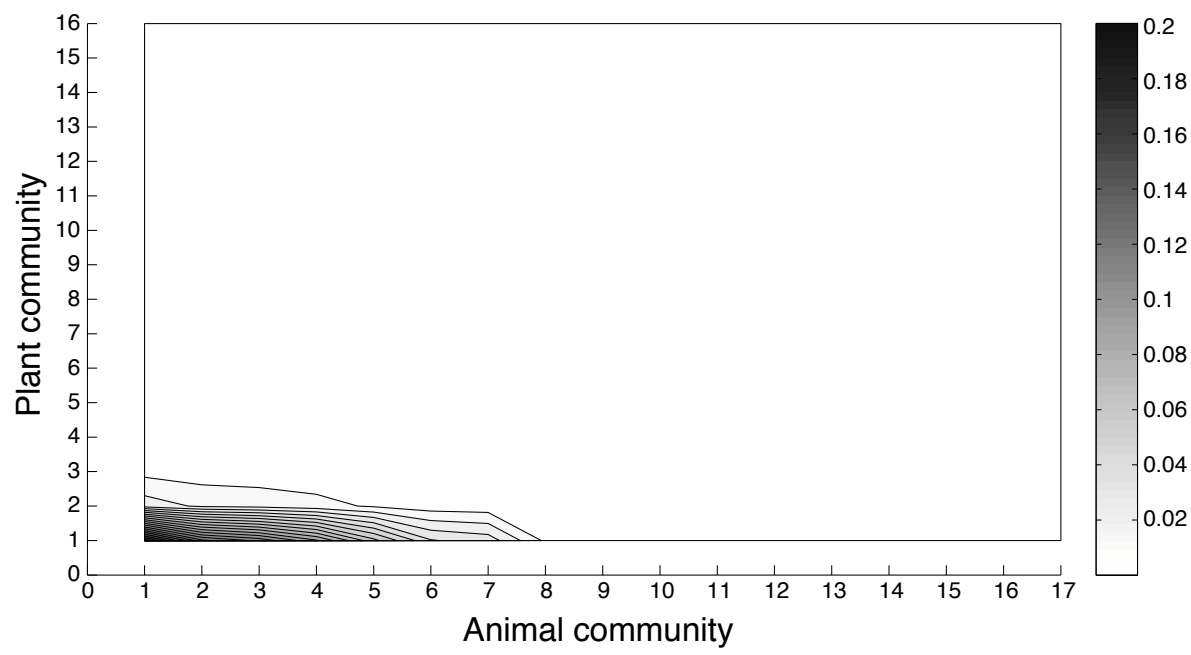

Figure 1. An example of a best-fit simulated counterpart of a real mutualistic network (Hato Ratón) and its RSA based interactions. The sub plot on the upper left represents the plant dominance diversity curve for the Hato Ratón plant community, solid lines indicate the real dominance diversity curve and the broken line represents the dominance diversity curve obtained by parameters of best ML fit for the Hato Ratón plant community, $\mathrm{m}$ (dispersal probability) is $0.99 ; \theta$ (fundamental biodiversity number) is 1.83 ; J (number of individuals) is 8499 ; and $s$ (number of species) is 16 . The sub plot on the upper right represents the animal dominance diversity curve for the Hato Ratón animal community, solid lines indicate the real dominance diversity curve and the broken line represents the dominance diversity curve obtained by parameters of best ML fit for the Hato Ratón animal community, $\mathrm{m}$ (dispersal probability) is 0.0081 ; $\theta$ (fundamental biodiversity number) is 3.72; J (number of individuals) is 7883; and s (number of species) is 17 . The lower subplot represents the probability contour plot of abundance-based interaction in such a neutral mutualistic community. The contours represent the super-generalist core with probability of interaction ranging from $0.02-0.2$.

and the higher end of the connectance range $(0.26-0.4)$ with lower number of species (15-100).

\section{Real mutualistic networks}

The studied mutualistic communities correspond to a longterm ecological study by one of the authors (PJ) with two plant-frugivore interaction networks, namely a high elevation Mediterranean pine forest composed of 33 frugivore species and 25 species of fruiting plants (Nava de las Correhuelas, Sierra de Cazorla, southeast Spain) and 17 frugivore species and 16 fleshy-fruit producing plants in a lowland Mediterranean scrubland (Hato Ratón, Parque Natural de Donana, southwest Spain) (Jordano 1988, 1994, 1995).

The abundance estimates for frugivores were derived from line transect counts involving direct census (Emlen 1971). The plant abundance data were obtained from transects counts of fruit production that actually indicate the availability of fruits of different species to the frugivore consumers (Jordano 1993). The interaction data were obtained along independent feeding records by direct observation of the fruiting plants. These types of independent estimates of abundance and the interaction matrix are difficult to obtain, but are needed for a robust test that avoids circularity arising from abundance estimates derived directly from the interaction frequency data. The dataset contains records from $>200 \mathrm{~km}$ line transect census of frugivore abundances (1987-2001) and 7010 feeding records of frugivores visiting fruiting plants for Nava de las Correhuelas network. For the Hato Ratón network, abundance data (1981-1983) were obtained similarly (Jordano 1988). Fruit-frugivore interaction data were obtained from mist-netting birds and the analysis of fecal samples to estimate the use of fruits (Jordano 1988), totalling 3404 records. A feeding record entails the observation of a frugivore, handling and eventually ingesting a fruit (Snow and Snow 1988). The study period for both areas encompassed the whole year cycle (Jordano 1988, 1993, 1994, 1995).

For these real communities maximum log-likelihood (ML) estimates of parameters derived from the neutral theory were also calculated separately for frugivores and plants (Table 1) using improved likelihood method (Etienne 2005). Using these parameters for plants and frugivores of real mutualistic communities, we generated DLM distributions and created 100 simulated neutral counterparts of real mutualistic networks in the same 
Table 1. Parameter estimates under the neutral theory for two real frugivore communities, obtained with the exact likelihood method, along with all local maxima if there is more than one local maximum. The parameters corresponding to the lowest maximum are denoted by $\theta_{\mathrm{l}}, \mathrm{m}_{\mathrm{l}}$. The log-likelihoods are also shown, the lowest log-likelihood is denoted by loglik .

\begin{tabular}{|c|c|c|c|c|c|c|}
\hline Communities & $\theta$ & $\mathrm{m}$ & Loglik & $\theta_{\mathrm{L}}$ & $\mathrm{m}_{\mathrm{L}}$ & Loglik $_{\mathrm{L}}$ \\
\hline Correhuelas plant community & 2.76 & 0.99 & -87.3 & 15.77 & 0.14 & -87.4 \\
\hline Correhuelas bird community & 32.5 & 0.0023 & -89.5 & 6.55 & 0.0063 & -89.7 \\
\hline Hato Ratón plant community & 1.83 & 0.99 & -55.3 & 9.45 & 2.15 & -60.6 \\
\hline Hato Ratón bird community & 3.72 & 0.0081 & -79.8 & none & none & none \\
\hline
\end{tabular}

manner as we generated NMN from a range of theoretical values of $\mathrm{m}$ and $\theta$. These simulated counterparts of real mutualistic networks were also subjected to nestedness analysis. The abundance for plants in these two communities were obtained by assuming that the number of fruits of a given species is positively correlated with the number of individuals of a species. Though the assumption is arguably not true for all species, it is essentially a first step to extend the neutral theory from a single trophic level to multiple trophic levels. Besides, total fruit availability is the best estimator of realized abundance in terms of interaction patterns than the raw number of individuals.

\section{Nestedness analysis}

A visual diagram of nestedness could be made by arranging all species in decreasing order of number of interactions. In a perfectly nested matrix all such interactions will be found towards the upper-left corner of such a matrix. We quantified nestedness $\mathrm{N}$ using the matrix temperature $\mathrm{T}$. The temperature of the interaction matrix is calculated as the ratio of sum of squared deviations from the boundary line (isocline of a maximally packed matrix) of unexpected presences and absences and the maximum value possible for an interaction matrix, multiplied by 100 (Atmar and Patterson 1993). We followed Bascompte et al. (2003) and defined the degree of nestedness in terms of matrix temperature

$\mathrm{N}=\frac{100-\mathrm{T}}{100}$

Second, we used null models to test if the observed degree of nestedness differs from random assignment based on a process of interest (Bascompte et al. 2003). The null model I (Atmar and Patterson 1993) assumes that each randomly assigned pair of plant and animal interacts with constant probability, $\mathrm{C}$, in which $\mathrm{C}$ is the connectance, i.e. the proportion of interactions actually observed in the network. Null model II (Bascompte et al. 2003) assumes that the probability that a plant interacts with an animal depends on the observed number of interactions of both species, such that the probability of cell occupancy is given as:

$\frac{1}{2} \mathrm{P}_{\mathrm{ri}}+\mathrm{P}_{\mathrm{cj}}$

where $\mathrm{P}_{\mathrm{ri}}$ is the fraction of presence denoting cells computed for rows and $\mathrm{P}_{\mathrm{cj}}$ is the fraction of presence denoting cells in columns.

We also estimated the relative nestedness (Bascompte et al. 2003), a measure of how nested is the matrix when compared with the mean expected nestedness under a given null model:

$\mathrm{N}^{*}=\frac{\mathrm{N}-\overline{\mathrm{N}}_{\mathrm{r}}}{\overline{\mathrm{N}}_{\mathrm{r}}}$

where $\mathrm{N}^{*}$ is relative nestedness, $\mathrm{N}$ is nestedness of a given mutualistic community and $\overline{\mathrm{N}}_{\mathrm{r}}$ is the average nestedness of 1000 replicates of the null model. We calculated the relative nestedness as compared to null models I and II terming it as relative nestedness I and relative nestedness II respectively. Relative nestedness allows a comparison of networks of varied sizes and connectances (Bascompte et al. 2003). Nestedness analysis was performed using the freely available software Aninhado (Guimarães and Guimarães 2006).

\section{Estimating the quantitative contribution of abundance to nestedness}

We also estimated the relative contribution of abundance to nestedness, i.e., to what extent nestedness varies when abundance variation across species is controlled. This method sorts out problems with standard statistical approaches such as partial correlations.

The nestedness temperature calculator (NTC) rearranges the matrix to maximize nestedness. This rearrangement is known as packing. In the proposed approach, we noted that if a given factor such as RSA totally explains nestedness, we should expect that if we organize both animal and plant species from the most to the least abundant, that would coincide with the packing performed by the NTC. That is the recorded value for nestedness $\mathrm{N}_{\mathrm{ab}}$ after packing for abundance would equal the maximum nestedness value $(\mathrm{N})$ after the packing procedure, performed by the NTC. Therefore, the ratio $\frac{\mathrm{N}_{\mathrm{ab}}}{\mathrm{N}}$ would vary from zero (abundance does not explain any aspect of nested assemblage) to one (abundance totally explains nestedness). Thus $\frac{\mathrm{N}_{\mathrm{ab}}}{\mathrm{N}}$ quantifies to what extent, observed nestedness is explained by abundance (RSA). To analyze if the contribution of RSA is statistically significant, we compare the value $\mathrm{N}_{\mathrm{ab}}$ to an empirically-derived null distribution $\mathrm{N}_{\mathrm{r}}$ obtained from an ensemble of matrices in which the organization of rows and columns was determined from the null models. We use this approach on both real mutualistic networks and its simulated counterparts.

This approach was also used to see if relative effective abundance (abundance corrected by the intensity of frugivory) explains the nested pattern in real communities 
more than abundance alone. Thus this approach exploits the possibility of calculating the temperature and thus nestedness of matrices in different arrangements and comparing them with maximally packed matrices (see Fig. 2 on how the matrix can have different arrangements).

\section{Disentangling the neutral processes shaping nestedness}

Extending the concept of neutrality to multiple trophic levels involves two independent parts, SAD generated by neutral theory and multiplicative abundance-correlated interaction probability. To disentangle the relative contribution of SAD and the assumption of random encounters based on abundance, we also created comparable networks from different SAD combinations (DLM, log normal, uniform) and the assumption of abundance based random encounters. Nestedness analysis was performed on this small subset of networks. This analysis is discussed to stress that other SAD combinations of plants and animals apart from DLM SAD, when interacting on the basis of an abundancecorrelated probability, will also give rise to high values of nestedness; though deviating differently from perfect nestedness.
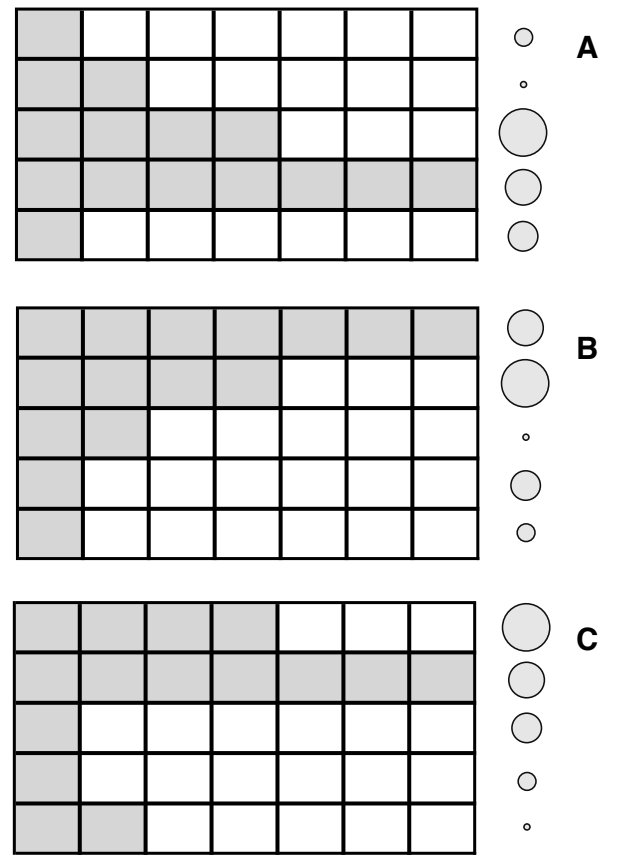

Figure 2. Matrices arranged by rows on the basis of a given ecological attribute (e.g. relative abundance or abundance weighted by intensity of dependence, here called effective relative abundance). Matrix A, B, and $\mathrm{C}$ show a random ordering, a maximally packed matrix maximizing nestedness, and a matrix ordered by a given ecological attribute, respectively. The area of circle represents the magnitude of the ecological attribute. As ordered by rows in the figure, we can also arrange these matrices by columns in different arrangements. The temperature of different arrangements is different and this forms the basis of the method to quantify the effect of given attribute on nestedness.

\section{Factors modulating the effects of abundance}

We also investigated how forbidden links (Jordano et al. 2003) and intensity of frugivory (Jordano and Schupp 2000) modulate the effects of species abundance on nestedness. Forbidden links are interactions that cannot occur due to constraints for a given pair of species. Most of the forbidden interactions are forbidden due to phenological uncoupling, size restrictions, or accessibility issues (Jordano et al. 2003). The intensity of frugivory is the relative importance of fruits for the diet of a given frugivore, markedly affecting seed dispersal. For example, an abundant but not highly frugivorous bird species might establish few interactions while several plant species might depend on a rare but strongly frugivorous species. These factors were chosen to better explain the nested pattern in real communities due to availability of quality data and the simplicity of evaluation for these factors.

We performed numerical simulations to explore the potential consequences of different frequencies of forbidden links in modulating abundance-generated nestedness in neutral networks and simulated counterparts of real networks. Besides simulating different frequencies of forbidden links in neutral networks of different shapes and sizes, we also calculated the frequencies of probable forbidden links in two real communities (Hato Ratón: $48 \%$ and Correhuelas: $35 \%)$. We introduced varying frequencies of forbidden links in simulated counterparts of these real networks and explored how much abundance explains nestedness in these simulated counterparts with different frequencies of forbidden links (Fig. 4). The contrasting curve for two communities (pattern for Hato Ratón decrease and then increase and pattern for Correhuelas increase and then decrease) could be due to differences in the real proportion of forbidden links in these two communities. A reasonable estimate of forbidden links in Hato Ratón is $30-50 \%$, whereas in Correhuelas, a conservative estimate of forbidden links is $20-38 \%$. The contrasting behavior of the curves could also be due to matrix size effects. This needs to be tested more rigorously but forbidden links seem to modulate the effect of abundance in a complex way. We also calculated relative nestedness of the simulated counterparts with identical frequency of forbidden links as calculated in real networks.

Calculation of forbidden links has its own limitations, e.g. not all frugivores consume whole fruit, and unobserved interactions may be due to sampling bias. Rarefaction analysis of the feeding record data and the faecal sample data in Jordano et al. (2008) have shown that the sampling of interactions in these communities is robust.

For our real mutualistic networks we have data on the intensity of frugivory as the proportion of fruits in a frugivore species diet. We quantified the relative contribution of fruit material to the diet of frugivore species as determined by dietary analysis of fecal samples (Jordano 1988). This value hereafter known as relative intensity of frugivory was not used to get quantitative networks or continuous values, but was used to estimate the effective abundance under modulation of intensity of frugivory in a very simple way:

$\mathrm{E}_{\mathrm{a}}=\mathrm{R}_{\mathrm{a}} \mathrm{P}_{\mathrm{f}}$ 
where $E_{a}$ is the effective relative abundance of frugivore species $a, R_{a}$ is the relative abundance of frugivore species a and the $P_{f}$ is the proportion of fruits in the diet of the frugivore a. Effective relative abundance, $\mathrm{E}_{\mathrm{a}}$ is a simple abundance data weighted by the reliance on fruit food for each species.

We used $\mathrm{E}_{\mathrm{a}}$ of frugivores to arrange the Correhuelas and Hato Ratón matrices in the same way, as we arranged a given matrix according to abundance of frugivores and fruiting plants (Fig. 2 for possible arrangements). We calculated a $\mathrm{N}$ for this arrangement (hereafter $\mathrm{N}_{\text {fab}}$ ). We, then compared $\mathrm{N}_{\mathrm{fab}}$ with $\mathrm{N}_{\mathrm{ab}}$, generated by arranging the matrix using solely the unweighted abundance data. If intensity of frugivory modulates the effects of abundance on nestedness, we should expect effective abundance to explain a larger fraction of the nested pattern than abundance alone and therefore $\mathrm{N}_{\mathrm{fab}}>\mathrm{N}_{\mathrm{ab}}$. However, the observed difference between $\mathrm{N}_{\mathrm{fab}}$ and $\mathrm{N}_{\mathrm{ab}}$ may be generated by chance (i.e. by randomly associating an intensity of frugivory with a relative abundance). We used re-sampling methods (Manly 1998) and calculated the empirical distribution of the difference between $\mathrm{N}_{\mathrm{fab}}$ and $\mathrm{N}_{\mathrm{ab}}$ by assuming a random association between intensity of frugivory and relative abundance, i.e., the intensity of frugivory is randomized among the species (10000 re-samplings). If the observed difference between $\mathrm{N}_{\mathrm{fab}}$ and $\mathrm{N}_{\mathrm{ab}}$ is significantly higher than expected by chance, a significant fraction of nestedness is explained by considering the additional effects of intensity of frugivory over abundance, than abundance alone.

\section{Results}

Abundance-based interactions lead to nearly perfect nestedness in neutral mutualistic networks (nestedness \pm SE equal to $0.994 \pm 0.005$ for 20000 neutral mutualistic networks). These neutral networks were significantly more nested than expected using both null models I and II. Not surprisingly, in all cases the nested pattern is completely explained by abundance $\left(\frac{\mathrm{N}_{\mathrm{ab}}}{\mathrm{N}}=1\right)$. In real-simulated neutral mutualistic networks, we also observe the similar nearly perfect nestedness completely related to abundance $\left(\frac{\mathrm{N}_{\mathrm{ab}}}{\mathrm{N}}=1.00, \mathrm{P}<0.001\right)$

The comparison between neutral mutualistic networks and real mutualistic networks shows that they differ in at least two aspects of nested patterns. First, Bascompte et al. (2003) have shown that real mutualistic networks are not perfectly nested (nestedness \pm SE equals to $\mathrm{n}=0.844 \pm$ 0.043 for seed dispersal and $\mathrm{N}=0.853 \pm 0.047$ for pollination). Second, although abundance explains a significant portion of nestedness in real networks, yet it does not explain the nestedness pattern completely in real networks, $\left(\frac{\mathrm{N}_{\mathrm{ab}}}{\mathrm{N}}=0.60, \mathrm{P}=0.004\right)$ for Correhuelas net- work, and $\left(\frac{\mathrm{N}_{\mathrm{ab}}}{\mathrm{N}}=0.67, \mathrm{P}<0.001\right)$ for Hato Ratón
network.

The differences between the degree of nestedness in real and theoretical networks is partially explained by forbidden links added to theoretical networks. Adding forbidden links leads to smaller values of relative nestedness (Fig. 3) comparable to relative nestedness values in real networks studied in Bascompte et al. (2003). When we introduced the calculated real frequency of forbidden links (Hato Ratón: $48 \%$ and Correhuelas: $35 \%$ ) in the simulated counterparts of these real networks, we found that relative nestedness of simulated counterparts fell in the range of relative nestedness of real networks. This analysis yield us results qualitatively similiar to our analysis of large dataset of real and neutral mutualistic networks (Fig. 3). Moreover, in the presence of forbidden links, abundance does not completely explain nested pattern and there is a constant fraction $(10 \%)$ of the nested structure that is not explained by abundance (Fig. 4). Therefore, forbidden links have a dual effect on nested patterns, reducing the overall nested pattern and modulating the effects of abundance on observed nestedness. However, forbidden links and abundance together did not completely explain the nested pattern in real communities either; suggesting that additional multiple effects are at work in a complex way in the modulation of perfect nestedness that arise only by neutral processes of community organization.

In fact, simply by weighting the abundance of frugivores by their intensity of frugivory, we significantly improve the fraction of nestedness explained (for Correhuelas network: $\frac{\mathrm{N}_{\mathrm{fab}}}{\mathrm{N}}=0.70$, a $15.85 \%$ increase, $\left.\mathrm{P}=0.009\right)$ and for Hato Ratón network: $\frac{\mathrm{N}_{\mathrm{fab}}}{\mathrm{N}}=0.72$, a $8.8 \%$ increase, $\mathrm{P}=0.037$ ) (Fig. 5).

\section{Discussion}

\section{Neutral-niche interplay}

By extending the neutral theory of biodiversity (Bell 2001, Hubbell 2001) from a single trophic community to an ecological network of mutualists, we illustrate that a nearly perfect nested organization emerges from such neutral mutualistic networks. When comparing the relative nestedness of neutral mutualistic networks with real networks (thus accounting for differences in connectance and number of species) we find that the relative nestedness of such neutral mutualistic networks is still way higher than in real communities. Using a novel approach with high quality data, we quantify the effect of abundance on nestedness and find that abundance plays a significant role in explaining the nestedness observed in real communities. In the realsimulated neutral mutualistic networks abundance completely explains the observed nestedness. Through theoretical predictions and real field data analysis, we show that besides abundance, additional interaction-specific species attributes 


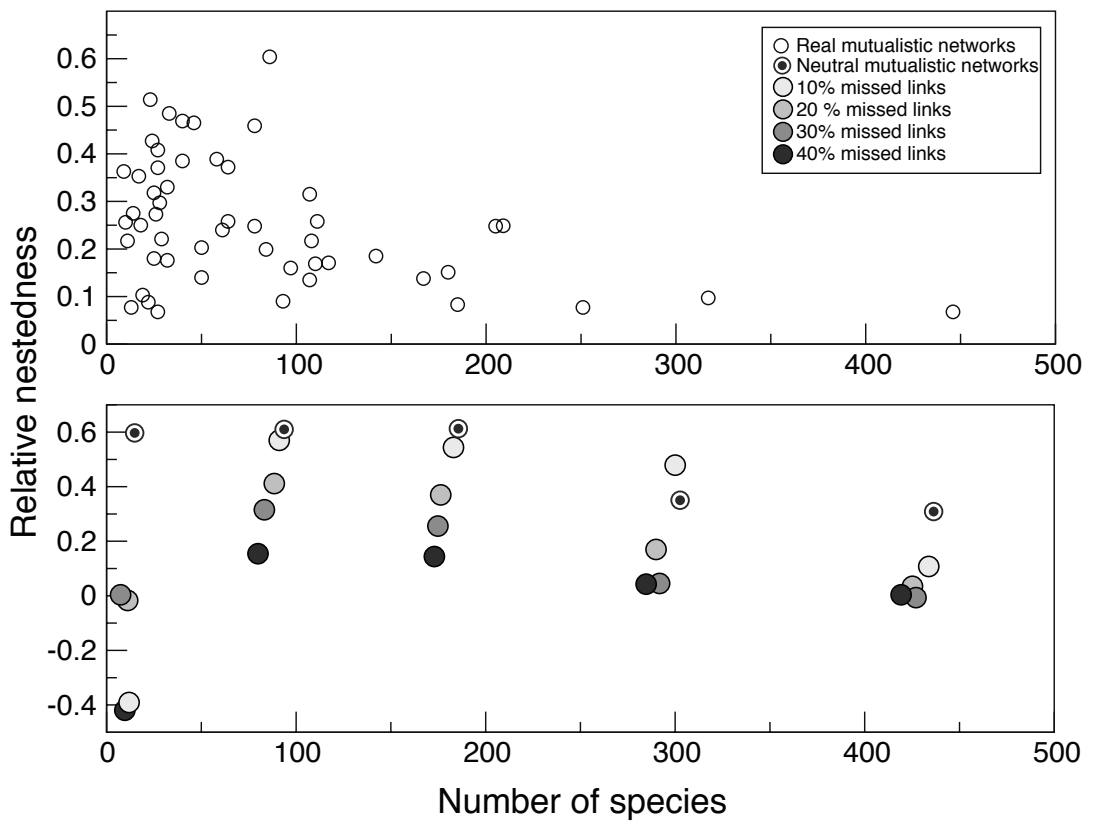

Figure 3. Relative nestedness values using null model II for the real communities analyzed in Bascompte et al. (2003) compared with neutral mutualistic communities consisting of interacting neutral plant community and animal community, $\mathrm{m}$ (dispersal probability) is 1 ; $\theta$ (fundamental biodiversity number) takes values 1, 10, 25, 50 and 100; J (number of individuals) is 1000. The upper subplot shows relative nestedness of real mutualistic networks and lower subplot shows average relative nestedness of 800 replicates of a neutral mutualistic network for each parameter combination and with variable proportions of forbidden links. Number of species in lower subplots is the average number of species in 800 replicates of neutral mutualistic networks. The results for relative nestedness using null model I were qualitatively similar.

(forbidden links, intensity of frugivory) influence the nested organization.

There is an intense ongoing debate in ecology about whether species-specific processes (niche/tradeoff/adaptation) or neutral processes shape community structure (Fargione et al. 2003). Here we show that real mutualistic networks are assembled by modulatory interaction-specific species traits (forbidden links, intensity of frugivory, and unknown traits) acting on neutral processes (abundancebased probability of interactions). Our results support the hypothesis that both neutral and niche based processes shape the community (Leibold and McPeek 2005), and point that assessing their relative contributions to community assembly will be a future challenge.

A recent study (Stang et al. 2006) had found that the number of interactions in a plant-pollinator web is determined by floral abundance and size constraints. Two other notable studies (Rezende et al. 2007a, Santamaria and Rodriguez-Girones 2007) had found that phenotypic complementarity could give rise to the nested structure.

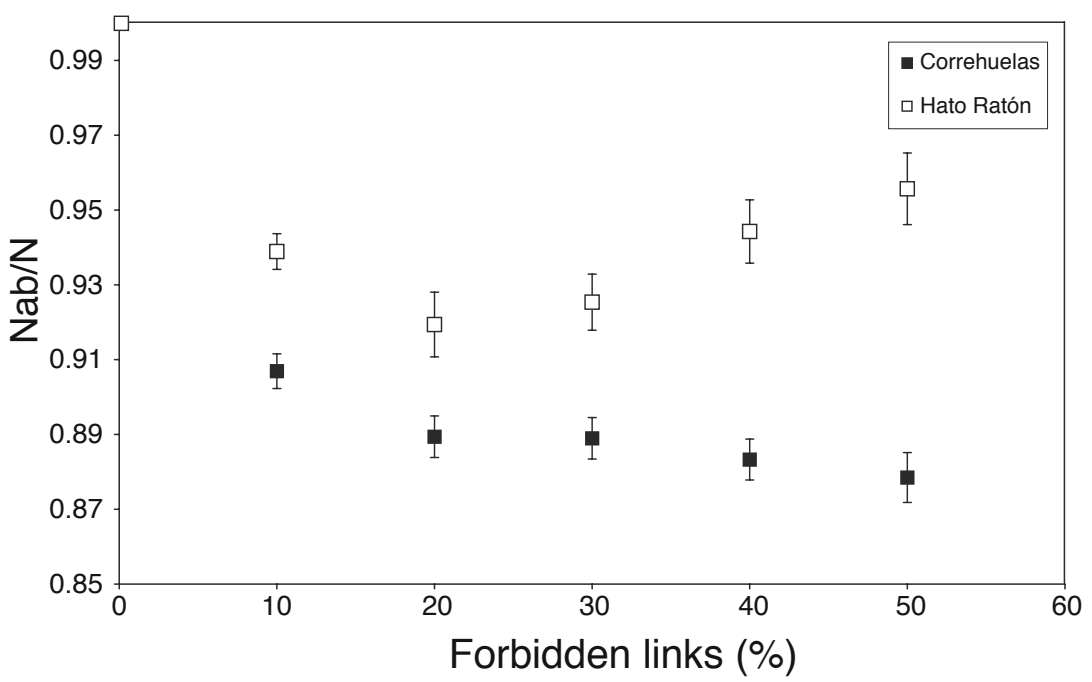

Figure 4. Relationship between the percentage of forbidden links in real-simulated neutral networks and the proportion of nested pattern accounted by abundance for the plant-frugivore networks of Nava de las Correhuelas (filled symbols) and Hato Ratón (open symbols). The error bars represent the standard deviation after 100 replicates of the networks analyzed. 


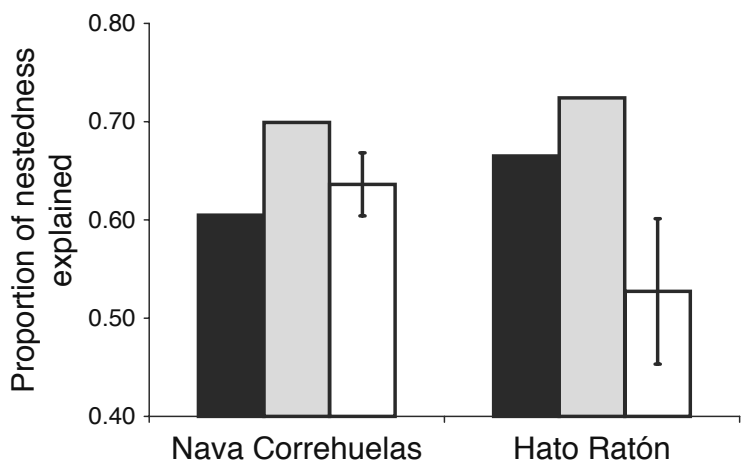

Figure 5. Bar graphs for two real communities depicting the quantitative estimate of the contribution of abundance and frugivory-intensity weighted abundance on nestedness. Black bars indicate the fraction of nestedness explained by abundance, grey bars indicate the fraction of nestedness explained by frugivoryintensity weighted abundance and white bars indicate the average fraction of nestedness explained by frugivory-intensity weighted abundance for networks in which frugivory was randomly distributed among species. Standard errors are also shown for these randomized networks. The effect of frugivory intensity on abundance is statistically significant when compared to resamplings (white bars).

Phylogeny had also been adduced as a determinant of network structure (Rezende et al. 2007b). Finally, a recent study had found that asymmetric interaction strength in quantitative mutualistic webs is positively correlated with species abundance, but species abundance alone could not explain the asymmetry observed (Vazquez et al. 2007). Our study adds to this set of recent papers by quantifying the important contribution of species abundance to the overall network patterns. Here we find a quantitative estimate of the relative effect of abundance and other ecological traits of species to the nested structure observed in real frugivore communities. RSA is an important contributor in explaining nestedness pattern, about $60-70 \%$ of nestedness pattermn is explained by abundance in two real communities. Moreover, about $30-40 \%$ of nestedness pattern is still unexplained in two real communities. Given, the initial result of neutral communities being highly nested, and analysis of other SAD types, we can say by confidence that any reasonable SAD type (DLM, log-normal) of plant and animal abundances, interacting on the basis of multiplicative process of probability, will result in far more nested communities than real communities. Thus there is an evidence of abundance modulating processes in real communities studied here. We should expect the effects of local abundance to be pervasive in interaction networks of generalized, free living animals and plants, where probability of interaction is expected to be correlated, to a variable degree, with abundance. Networks depicting more intimate associations between animals and plants (i.e. higher specificity of the interactions) should be less influenced by local abundance.

Thus, although RSA explains much of the nested pattern observed in real networks, it does not fully explain the lower values of nestedness in real networks, when compared to neutral networks. This study highlight the importance of taking into account both abundance and interactionspecific species traits when making conservation decisions regarding mutualistic communities. The approach proposed here can then be used to explore what is the relative contribution of these processes to the nested structure. For instance, local frugivore abundance has been shown to track local fruit supplies, while the eco-morphological traits of frugivores and their physiological characteristics, that determine efficient fruit consumption (e.g. body mass, bill morphology, digestive function) are more directly linked to phenotypic variation and evolutionary history (Moermond and Denslow 1985, Jordano 2000). Ecologically-labile characteristics such as local abundance seem extremely important in assembling mutualistic interactions among free-living species that are based on high diversity and low specificity of the interactions; yet evolutionary convergence based on species-specific attributes can complement these processes by driving more specialized interactions (Thompson 2005, Rezende et al. 2007b). Our data support this key modulating effect of the two processes.

\section{Abundance and nestedness}

RSA is an important contributor in explaining nestedness pattern, about $60-70 \%$ of nestedness pattermn is explained by abundance in two real communities. Almost $40 \%$ of the nestedness pattern in real communities is not explained by abundance. This raises an important point that real observed nested structure of mutualistic networks is a result of the interplay of several ecological processes (neutral assembly rules and both known and unknown speciesspecific traits). To dissect all the mechanisms giving rise to the complex patterns of mutualistic networks, such as their universal nested structure (Bascompte et al. 2003), we require innovative empirical and theoretical approaches (Shipley et al. 2006, Adler et al. 2007).

This work differs from previous null model analyses of specialization in mutualistic networks (Vázquez and Aizen 2003) in three distinct ways. Firstly we use the dispersal limited multinomial (DLM) distribution predicted by neutral theory in place of $\log$ normal distributions to set up the interactions, but see the next sub section. Secondly, we use estimates of species abundance which are independent of the actual interaction data. Finally we add several ecological variables in concordance with species abundance and quantify their effects on nestedness. Moreover our work successfully extends neutral theory of single trophic community to multiple trophic levels and thus is a step towards bridging the niche-neutral debate.

\section{SAD and neutral theory of ecological interactions}

Species-abundance distribution (SAD) is one of the oldest and most famous laws in ecology. Recently, McGill et al. (2007) have advocated the integration of such SAD within an ecological framework. Here we use SAD obtained from neutral theory to show that a universal pattern of mutualistic communities, namely nestedness, can emerge from abundance based interactions among mutualists SADs. An extended analysis also showed that high values of nestedness can emerge from abundance based interactions of other realistic SAD combinations of plants and animals (log-normal), though differing in deviations from 
perfect nestedness. This analysis highlights the role of multiplicative process of abundance based probabilities of interactions as a chief component of the neutral processes giving rise to nestedness independent of SAD types. The approach can be used for all bipartite webs (for e.g. predator-prey networks) in neutral theory context.

An extension of neutral theory of unitrophic communities towards a neutral theory of ecological interaction throws as many challenges as opportunities. The challenges that are evident from this work are:

a. What should be the currency of abundance when extending the effect of abundance on interaction? Here we have used the number of fruits as a currency of realized abundance of plants for interaction with birds opposed to the number of individual tree or plants as a currency of abundance in unitrophic neutral theory. This challenge is probably evident in our ML estimation of neutral theory parameters for real communities where we find the dispersal probability of plants as much higher than birds. This is a seemingly implausible result but could have arisen because of our use of realized abundance (a lower sample size could cause biases).

b. There are a large number of SAD types and SAD models and a strong inference is needed to reject a particular SAD type or SAD model. Thus there is a need of more empirical data similar to the data used in this study for a strong inference.

c. How to incorporate more than two trophic levels and more than a single type of interaction? What if we also have insects now in addition to fruiting plants and birds? The birds can consume these insects, but on the other hand insects are also herbivores and pollinators for plants. How can we extend SAD and neutral theory in such a context?

\section{Towards a new test of non-neutrality/niche differentiation}

The approach used here, i.e. taking into consideration a universal pattern of mutualistic community namely nestedness, and interaction specific species traits can be used as a test to show that to what extent our data deviates from neutrality. Thus, the challenge is, can a signal of non neutrality/niche-differentiation be picked from the SAD's of related trophic level and Nestedness values/interactionspecific species traits? If yes, how strong is this signal? Can such a test identify the most important species interaction or non-interaction from a perspective of niche differentiation (deviation from neutrality)? We hypothesize, thus that the unexpected presence/absence of interaction that results in deviation of perfect nestedness could be a signature of non neutrality/niche differentiation.

The continuing developments in formulation of neutral theory (Etienne 2007, Rosindell and Cornell 2007) and the healthy debate surrounding it will ensure that neutral theory will be a useful concept in community ecology (Alonso et al. 2006). A future integration of sampling theories (neutral theory) and network ecology can result in a sampling theory for ecological networks of species interactions.
This will facilitate the establishment of SAD as the master pattern in ecology and its integration within an ecological framework McGill et al. (2007).

Acknowledgements - We thank M. A. Fortuna and P. Guiniaraes for technical help and D. Alonso for discussion. We also thank J. Carnicer, A. Valido, E. Rezende and R.S. Etienne for very helpful comments on previous drafts of this paper. This work was funded by the European Heads of Research Councils, the European Science Foundation, and the EC Sixth Framework Programme through a EURYI (European Young Investigator) Award (to J.B.). PRG was supported by FAPESP. Work by PJ and JB was also funded by grants CGL2006-00373 from the Spanish Ministerio de Ciencia y Tecnologia (MEC)and RNM-305 from the Junta de Andalucia. The Consejeria de Medio Ambiente kindly authorized our field work in Parque Natural de las Sierras de Cazorla, Seguray las Villas.

\section{References}

Adler, P. B. et al. 2007. A niche for neutrality. - Ecol. Lett. 10: 95-104.

Alonso, D. et al. 2006. The merits of neutral theory. - Trends Ecol. Evol. 21: 451-457.

Atmar, W. and Patterson, B. D. 1993. The measure of order and disorder in the distribution of species in fragmented habitat. - Oecologia 96: 373-382.

Bascompte, J. et al. 2003. The nested assembly of plant-animal mutualistic networks. - Proc. Natl Acad. Sci. 100: 9383-9387.

Bell, G. 2001. Neutral macroecology. - Science 293: 2413-2418.

Dupont, Y. L. et al. 2003. Structure of a plant-flower-visitor network in the high-altitude sub-alpine desert of Tenerife, Canary Islands. - Ecography 26: 301-310.

Emlen, J. T. 1971. Population densities of birds derived from transect counts. - Auk 88: 323-342.

Etienne, R. S. 2005. A new sampling formula for neutral biodiversity. - Ecol. Lett. 8: 253-260.

Etienne, R. S. 2007. A neutral sampling formula for multiple samples and an exact test of neutrality. - Ecol. Lett. 10: 608618.

Etienne, R. S. and Olff, H. 2004. A novel genealogical approach to neutral biodiversity theory. - Ecol. Lett. 7: 170-175.

Etienne, R. S. et al. 2007. The zero-sum assumption in neutral biodiversity theory. - J. Theor. Biol. 248: 522-536.

Fargione, J. et al. 2003. Community assembly and invasion: an experimental test of neutral versus niche processes. - Proc. Natl Acad. Sci. 100: 8916-8920.

Gotelli, N. and McGill, B. J. 2006. Null versus neutral models: what's the difference? - Ecography 29: 793-800.

Guimarães, P. R. and Guimarães, P. 2006. Improving the analyses of nest-edness for large sets of matrices. - Environ. Modell. Software 21: 1512-1513.

Guimarães, P. R. et al. 2006. Asymmetries in specialization in antplant mutualistic networks. - Proc. R. Soc. Lond. B 273: 2041-2047.

Guimarães, P. R. et al. 2007. The nested structure of marine cleaning symbiosis: is it like flowers and bees? - Biol. Lett. 3: 51-54.

Hubbell, S. P. 2001. The unified neutral theory of biodiversity and biogeography. - Princeton Univ. Press.

Jordano, P. 1987. Patterns of mutualistic interactions in pollination and seed dispersal: connectance, dependence asymmetries, and coevolution. - Am. Nat. 129: 657-677.

Jordano, P. 1988. Diet, fruit choice and variation in body condition of fru-givorous warblers in mediterranean scrubland. - Ardea 76: 193-209. 
Jordano, P. 1993. Geographical ecology and variation of plantseed disperser interactions: southern Spanish junipers and frugivorous thrushes. - Plant Ecol. 107: 85-104.

Jordano, P. 1994. Spatial and temporal variation in the avianfrugivore assemblage of prunus mahaleb - patterns and consequences. - Oikos 113: 174-184.

Jordano, P. 1995. Frugivore-mediated selection on fruit and seed size - birds and st lucies cherry, Prunus mahaleb. - Ecology 76: 2627-2639.

Jordano, P. 2000. Fruits and frugivory. - In: Fenner, M. (ed.), Seeds: the ecology of regeneration in plant communities. Commonwealth Agric. Bureau Int., pp. 125-166.

Jordano, P. and Schupp, E. W. 2000. Seed disperser effectiveness: the quantity component and patterns of seed rain for Prunus mahaleb. - Ecol. Monogr. 70: 591-615.

Jordano, P. et al. 2003. Invariant properties in coevolutionary networks of plant-animal interactions. - Ecol. Lett. 6: 69-81.

Jordano, P. et al. 2006. The ecological consequences of complex topology and nested structure in pollination webs. - In: From specialization to generalization in plant-pollinator interactions. - Univ. of Chicago Press, pp. 173-199.

Jordano, P. et al. 2008. Redes complejas de interacciones plantaanimal. - In: Medel, R. et al. (eds), Interacciones plantaanimal y la conservation de la biodiversidad. CYTED, in press.

Leibold, M. A. and McPeek, M. A. 2005. Coexistence of the niche and neutral perspectives in community ecology. - Ecology 87 : 1399-1410.

Lewinsohn, T. M. et al. 2006. Structure in plant-animal interaction assemblages. - Oikos 113: 174-184.

Manly, B. 1998. Testing for latitudinal and other body-size gradients. - Ecol. Lett. 1: 104-111.

McGill, B. J. et al. 2007. Species abundance distributions: moving beyond single prediction theories to integration within an ecological framework. - Ecol. Lett. 10: 995-1015.

Moermond, T. and Denslow, J. 1985. Neotropical avian frugivores: patterns of behavior, morphology, and nutrition, with consequences for fruit selection. - In: Buckley, P. et al. (eds), Neotropical ornithology. Ornithol. Monogr. no. 36. Am. Ornithol. Union, pp. 865-897.

Ollerton, J. et al. 2003. The pollination ecology of an assemblage of grassland Asclepiads in South Africa. - Ann. Bot. 92: 807834.

Ollerton, J. et al. 2007. Finding NEMO: nestedness engendered by mutualistic organization in anemone fish and there hosts. - Proc. R. Soc. Lond. B 274: 591-598.

Rezende, E. L. et al. 2007a. Effects of phenotypic complementarity and phylogeny on the nested structure of mutualistic networks. - Oikos 116: 1919-1929.

Rezende, E. L. et al. 2007b. Non-random coextinctions in phylogenetically structured mutualistic networks. - Nature 448: 925-928.

Rosindell, J. L. and Cornell, S. J. 2007. Species-area relationships from a spatially explicit neutral model in an infinite landscape. - Ecol. Lett. 10: 586-595.

Santamaria, L. and Rodriguez-Girones, M. A. 2007. Linkage rules for plant-pollinator networks: trait complementarity or exploitation barriers? - PLoS Biol. 5: e31.

Shipley, B. et al. 2006. From plant traits to plant communities: a statistical mechanistic approach to biodiversity. - Science 314: 812.

Snow, B. and Snow, D. 1988. Birds and berries: a study of an ecological interaction. - Poyser.

Stang, M. et al. 2006. Size constraints and flower abundance determine the number of interactions in a plant-flower visitor web. - Oikos 112: 111-121.

Thompson, J. N. 2005. The geographic mosaic of coevolution. - Univ. of Chicago Press.

Vázquez, D. P. and Aizen, M. A. 2003. Null model analyses of specialization in plant-pollinator interactions. - Ecology 84: 2493-2501.

Vazquez, D. P. et al. 2005. Species abundance and the distribution of specialization in host-parasite interaction networks. - J. Anim. Ecol. 74: 946-955.

Vazquez, D. P. et al. 2007. Species abundance and asymmetric interaction strength in ecological networks. - Oikos 116: $1120-1127$. 